\title{
Authors' Reply to Cerejeira: "Association of Early Systemic Corticosteroid Therapy with Mortality in Patients with Stevens-Johnson Syndrome or Toxic Epidermal Necrolysis: A Retrospective Cohort Study Using a Nationwide Claims Database"
}

\author{
Kojiro Morita $^{1}$ (1) $\cdot$ Hideo Yasunaga ${ }^{1}$
}

Published online: 12 September 2019

c) Springer Nature Switzerland AG 2019

We thank Dr. André Cerejeira for his interest in our study $[1,2]$. His main concern is about propensity-score matching (PSM), which was used in our study. His comments are based on a political scientist's criticism of PSM in a previous report [3]. We acknowledge that there are arguments by statisticians both for and against PSM. We are not in a position to make any comments on a statistician's criticism of a particular statistical method; however, there is general agreement that PSM is the most popular method for causal analysis in observational studies, which is why we used it in our study.

We also conducted inverse probability of treatment weighting (IPTW), which is a propensity score analytical method. The previous criticism of PSM does not apply to other propensity score analytical methods (including IPTW, regression adjustment, and stratification) [2]. Our analysis confirmed that the PSM and IPTW results were consistent.

This reply refers to the comment available online at https://doi. org/10.1007/s40257-019-00472-4.

This reply refers to the article available at https://doi.org/10.1007/ s40257-019-00443-9.

Kojiro Morita

kojiromorita-tky@umin.ac.jp

1 Department of Clinical Epidemiology and Health Economics, School of Public Health, Graduate School of Medicine, The University of Tokyo, 7-3-1 Hongo, Bunkyo-ku, Tokyo 113-0033, Japan

\section{Compliance with Ethical Standards}

Funding Dr. Yasunaga's institution received funding from the Ministry of Health, Labour and Welfare, Japan.

Conflict of interest Kojiro Morita and Hideo Yasunaga have disclosed they have no potential conflicts of interest.

\section{References}

1. Cerejeira A. Comment on: "Association of early systemic corticosteroid therapy with mortality in patients with Stevens-Johnson syndrome or toxic epidermal necrolysis: a retrospective cohort study using a nationwide claims database". Am J Clin Dermatol. 2019. https://doi.org/10.1007/s40257-019-00472-4.

2. Morita K, Matsui H, Michihata N, Fushimi K, Yasunaga H. Association of early systemic corticosteroid therapy with mortality in patients with Stevens-Johnson syndrome or toxic epidermal necrolysis: a retrospective cohort study using a nationwide claims database. Am J Clin Dermatol. 2019;20(4):579-92.

3. King G, Nielsen R. Why propensity scores should not be used for matching. Polit Anal. 2019. https://doi.org/10.1017/pan.2019.11. 